\title{
Association between the expression of T-cadherin and vascular endothelial growth factor and the prognosis of patients with gastric cancer
}

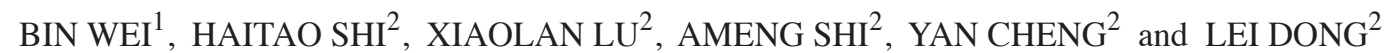 \\ ${ }^{1}$ Department of Gastroenterology, Xi'an No. 1 Hospital, Xi'an, Shaanxi 710002; ${ }^{2}$ Department of Gastroenterology, \\ The Second Affiliated Hospital of Medical School, Xi'an Jiaotong University, Xi'an, Shaanxi 710004, P.R. China
}

Received June 1, 2014; Accepted February 17, 2015

DOI: $10.3892 / \mathrm{mmr} .2015 .3592$

\begin{abstract}
T-cadherin has been identified as a tumor-suppressor gene in several types of cancer. The present study aimed to investigate the association of the expression of T-cadherin with angiogenesis, and to evaluate its prognostic value for patients with primary gastric cancer. Gastric cancer tissues and matched adjacent tissues from 166 patients receiving surgical resection were included in the present study. The expression of T-cadherin was detected using immunohistochemistry, western blotting and reverse transcription-quantitative polymerase chain reaction. The expression of vascular epidermal growth factor (VEGF) was detected using immunohistochemistry, and its association with the expression of T-cadherin was analyzed. In addition, the association between the expression of T-cadherin and clinicopathological features were analyzed. The mRNA and protein expression levels of T-cadherin were significantly lower in the gastric cancer tissue compared with the corresponding adjacent normal tissue $(\mathrm{P}<0.05)$. The expression of VEGF was not associated with the expression of T-cadherin in the gastric cancer tissue. The decreased protein expression of $\mathrm{T}$-cadherin correlated with smoking, larger tumor size (diameter, $>4 \mathrm{~cm}$ ), lymph node metastasis and a higher tumor-lymph node-metastasis stage $(\mathrm{P}<0.05$ or $\mathrm{P}<0.01)$. However, the expression of $\mathrm{T}$-cadherin was not correlated with gender, age, alcohol intake, Helecobacter pylori infection or differentiation $(\mathrm{P}>0.05)$. The multivariate analysis demonstrated that the expression of T-cadherin was an independent prognostic factor for the overall survival rate of patients with gastric cancer. This data suggested that the downregulation of T-cadherin may contribute to gastric cancer progression, representing a useful biomarker for predicting the
\end{abstract}

Correspondence to: Professor Xiaolan Lu, Department of Gastroenterology, The Second Affiliated Hospital of Medical School, Xi'an Jiaotong University, 157 Xiwu Road, Xi'an, Shaanxi 710004, P.R. China

E-mail: luxiaolan1967@163.com

Key words: gastric cancer, T-cadherin, metastasis, progress biological behavior and prognosis of gastric cancer. However, no significant association was observed between the expression of VEGF and T-cadherin.

\section{Introduction}

Gastric cancer remains the fourth most common type of cancer and the second most common cause of cancer-associated mortality worldwide (1). The majority of cases of gastric cancer are diagnosed at an advanced stage of development and, in patients no longer suitable for surgery or in cases of post-surgery recurrence, no effective treatment is currently available. Chemotherapy and radiotherapy often cause serious side effects as, in addition to causing cancer cell death, they affect normal tissue cells (2).

Cell-cell adhesion determines cell polarity and is involved in cell differentiation and the maintenance of tissue homeostasis. This cell-cell adhesion is disturbed during oncogenesis, resulting in changes in signaling, loss of contact inhibition and altered cell migration (3). There is increasing evidence implicating cell-cell adhesion molecules in cancer, as potent suppressors or as proto-oncogenic proteins (4). T-cadherin, also termed cadherin-13 (CDH13), is an atypical member of the cadherin superfamily, which is anchored to cell membranes via glycosylphosphatidylinositol (GPI) anchors, rather than transmembrane domains (5). Several previous studies have led to T-cadherin being considered a tumor suppressor, as it is frequently silenced in various types of cancer, including hepatocellular carcinoma (6), colon carcinoma (7), gallbladder carcinoma (8), melanoma (9), lung cancer (10) and breast cancer (11). Previous studies have demonstrated that the re-expression of T-cadherin may suppress cell proliferation, angiogenesis and invasiveness, increase sensitivity to apoptosis and decrease tumor growth (3).

Tang et al (12) revealed that the expression of T-cadherin was significantly reduced in tumor tissue samples compared with the adjacent normal tissue, and can be used as a biomarker for the progression and prognosis of gastric cancer. However, its tumor-suppressor mechanism in gastric cancer remains to be elucidated. Angiogenesis is required for invasive tumor growth and metastasis, and constitutes an important stage in the control of cancer progression $(13,14)$. A previous 
study demonstrated that the hyperexpression of T-cadherin in melanoma cells suppresses the growth of tumor masses and angiogenesis in vivo (15). However, Hebbar et al (16) demonstrated that T-cadherin promotes tumor angiogenesis in breast cancer. Therefore, the association between the expression of T-cadherin and angiogenesis remains inconsistent.

The present study aimed to evaluate the expression of $\mathrm{T}$-cadherin in gastric cancer, and analyze the association between the expression of T-cadherin and clinicopathological features in a larger number of patients. In addition, the association between the expression of T-cadherin and angiogenesis was investigated.

\section{Materials and methods}

Specimens and samples. Specimens of primary gastric cancer and matched adjacent normal tissue were obtained from 166 patients who underwent surgery at The Second Affiliated Hospital of College of Xi'an Jiaotong University (Xi'an, China), between 2010 and 2012. The mean patient age was 62.3 years (range, $41-82$ ). The tissue samples were frozen in liquid nitrogen immediately following resection and rinsing with phosphate-buffered saline (PBS), and were maintained at $-80^{\circ} \mathrm{C}$ until RNA and protein extraction. Data on the gender, age, smoking, alcohol intake, Helicobacter pylori $(\mathrm{Hp})$ infection, tumor size, lymph node metastasis and the tumor-lymph node-metastasis (TNM) stage of the patients with gastric carcinoma was also obtained. The International Union against Cancer TNM staging system (17) was used to classify the patients. Information on the survival rates of the 166 patients was followed up through written and telephone communication. The present study was approved by the Ethics Committee of The Second affiliated hospital of College of Xi'an Jiaotong University.

Reverse transcription-quantitative polymerase chain reaction $(R T-q P C R)$. Total RNA was extracted from the gastric cancer and adjacent normal tissue samples using a TRIzol kit (Gibco BRL, Grand Island, NY, USA), according to the manufacturer's instructions. The concentration of the total RNA was measured by spectrophotometry (ND-1000; Thermo Fisher Scientific, Waltham, MA, USA). The RNA was subsequently reverse-transcribed into cDNA using reverse transcriptase reagents (dNTP mixture and RNase free $\mathrm{dH}_{2} \mathrm{O}$; Takara Bio Inc., Otsu, Shiga, Japan). RT-qPCR was performed using the SYBR GreenI fluorescent dye method (SYBR Premix Ex Taq ${ }^{\mathrm{TM}}$ II; Takara Bio Inc.) and a Rotor Gene 3000 RT-qPCR apparatus (Qiagan, Shanghai, China). The following primers from (Beijing AuGCT Biotechnology Co., Ltd., Beijing, China) were used: T-cadherin, forward 5'-GATGTTGGCAAGGTAGTCGAT-3' and reverse 5'-GCT CCCTGTGTTCTCATTGAT and $\beta$-actin, forward 5'-ATCGTG CGTGACATTAAGGAGAAG-3' and reverse 5'-AGGAAGGA AGGCTGGAAGAGTG-3'. The expression of $\beta$-actin was used as an internal control to assess the relative expression of $\mathrm{T}$-cadherin. The PCR reaction was performed in a final volume of $20 \mu \mathrm{l}$, which contained $10 \mu \mathrm{l}$ SYBR Premix Ex TaqTM II, $1 \mu 1 \mathrm{cDNA}, 0.5 \mu 1$ of each primer and $8 \mu 1$ enzyme-free water. The PCR conditions were as follows: Predenaturing at $95^{\circ} \mathrm{C}$ for $3 \mathrm{~min}$, followed by 40 cycles of denaturation at $95^{\circ} \mathrm{C}$ for
$10 \mathrm{sec}$ and annealing/extension at $60^{\circ} \mathrm{C}$ for $30 \mathrm{sec}$. The amplification specificity was assessed by melting curve analysis. The PCR products were run on a $2 \%$ agarose gel (Sigma-Aldrich, St. Louis, MO, USA) stained with ethidium bromide (Shaanxi Pioneer Biotech Co., Ltd., Xi'an, China) and were observed to be 177 base pairs in size. The $2^{-\Delta \Delta C T}$ method (18) was used to calculate the relative expression levels of the target gene produced, using a Bio-Rad Real-Time analysis system (Bio-Rad Laboratories, Hercules, CA, USA).

Western blotting. The cytosolic protein extraction from the gastric cancer and adjacent normal tissuse samples were performed, as described previously. Samples of $50 \mu \mathrm{g}$ protein were mixed with gel loading buffer (Beyotime Institute of Biotechnology, Shanghai, China), boiled for $5 \mathrm{~min}$ and loaded onto 8 or $10 \%$ polyacrylamide gels (Shaanxi Pioneer Biotech Co.,Ltd.). Electrophoresis was performed and the proteins were transferred onto nitrocellulose membranes(Millipore,Billerica, MA, USA). Non-specific antibody binding was blocked by pre-incubation of the membranes with $1 \mathrm{X}$ Tris-buffered saline (TBS; Beijing ComWin Biotech Co., Ltd., Beijing, China), containing 5\% non-fat milk (Yili Group, Inner Mongolia, China) for $2 \mathrm{~h}$ at room temperature. The membranes were incubated overnight at $4^{\circ} \mathrm{C}$ with primary antibodies against human T-cadherin (rabbit polyclonal; 1:1,000; ABT121; Millipore) or $\beta$-actin (mouse monoclonal; 1:1,000; sc-47778; Santa Cruz Biotechnology, Inc., Santa Cruz, CA, USA) in 1X TBS, containing 5\% non-fat milk. Following washing with TBS for 5 min 3 times, the membranes were incubated with horseradish peroxidase-conjugated anti-rabbit immunoglobulin G (1:4,000; sc-2004; Santa Cruz Biotechnology, Inc.) for $2 \mathrm{~h}$ at room temperature. The bands were visualized using a SuperSignal Substrate Chemiluminescence kit (Millipore).

Immunohistochemistry. Paraffin-embedded (Sigma-Aldrich) tissue sections were de-waxed in xylene (Xi'an Chemical Reagent Factory, Xi'an, China) and rehydrated with a graded ethanol (Xi'an Chemical Reagent Factory) series. The slides used were from Wuhan Boster Biological Technology Ltd. (Wuhan, China). The tissue sections were subsequently treated with $3 \%$ hydrogen peroxidase (Zhongshan Jinqiao Bio., Beijing, China) for $15 \mathrm{~min}$ at room temperature, followed by incubation overnight at $4{ }^{\circ} \mathrm{C}$ with diluted primary antibodies against T-cadherin (1:100) or vascular epidermal growth factor (VEGF; mouse monoclonal; 1:100; sc-7269; Santa Cruz Biotechnology, Inc.,). The tissue sections were then incubated with secondary antibody. Specific reactivity was detected using a 3,3'-Diaminobenzidine Tetrahydrochloride kit (Zhongshan Jinqiao Bio.) and counterstained with hematoxylin (Sigma-Aldrich). The immunostaining was assessed by two independent pathologists in a blinded-manner. The slide used as a negative control was incubated with PBS rather than primary antibody. The positive controls used were cardiac muscle tissues known to exhibit high protein expression levels of T-cadherin. The cells were considered positive for the protein expression of T-cadherin when the cell membrane was stained. Each slide was evaluated in five randomly selected fields with an E100 microscope (Nikon, Tokyo, Japan) at a magnification of $x 400$, and 100-200 cells per field were counted. The immunohistochemical (IHC) scores comprise the product of 
Table I. Correlation between the expression of T-cadherin and clinicopathological variables in gastric cancer.

Expression of T-cadherin

Variable

Case (n)

Low (n)

High (n)

$\chi^{2}$

P-value

Gender

Male

108

52

56

Female

58

29

29

0.052

0.820

Age (years)

$<60$

$\geq 60$

80

42

39

38

47

0.848

0.357

Smoking

Yes

69

43

26

No

97

38

59

8.643

0.003

Alcohol consumption

Yes

99

45

No

67

36

54

31

1.096

0.295

Hp infection

Positive

122

58

Negative

44

23

64

21

0.290

0.590

Tumor size $(\mathrm{cm})$

$\leq 4$

99

39

60

$>4$

67

42

25

8.677

0.003

Differentiation

Well-moderate

63

103

25

56

38

47

Histopathological type

Intestinal

99

67

49

32

50

Diffuse

115

51

63

18

52

No

$79 \quad 28$

87

53

35

0.048

0.826

TNM stage

I-II stage

III-IV stage

\section{1}

34

0.066

Hp, Helicobacter pylori; TNM, tumor-lymph node-metastasis.

the scores of intensity ( 0 , no staining; 1 , weakly stained; 2 , moderately stained; 3 , markedly stained) and area $(0,<5 \% ; 1$, $5-25 \% ; 2,25-50 \% ; 3,>50 \%$ ) of the staining signals. Based on the expression of T-cadherin, the gastric cancer tissues were divided into two groups: A low T-cadherin expression group (IHC scores $\leq 3$ ) and the high T-cadherin expression group (IHC scores $>3$ ). The same method was used for the expression of VEGF, which was also divided into low and high expression groups.

Statistical analysis. All statistical analyses were performed using SPSS 13.0 software (SPSS, Inc., Chicago, IL, USA). The mRNA and protein expression levels of T-cadherin in the gastric cancer and matched adjacent tissues were analyzed using a paired sample t-test. The association

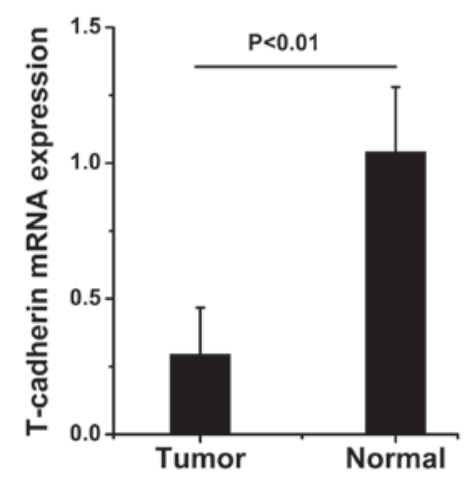

Figure 1. Reverse transcription-quantitative polymerase chain reaction analysis of the expression levels of T-cadherin in gastric cancer tissue samples. The relative mRNA expression of T-cadherin was significantly lower in the gastric cancer tissues compared with the corresponding normal tissues. The data are expressed as the mean \pm standard deviation; $\mathrm{P}<0.01$. 


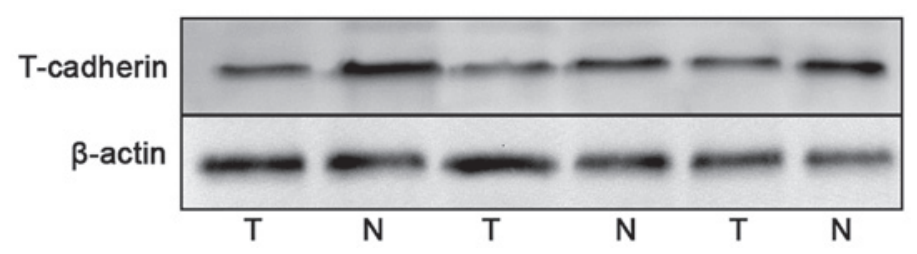

Figure 2. Protein expression levels of T-cadherin in gastric cancer, determined by western blotting. The protein expression levels of T-cadherin were markedly decreased in gastric tumor tissues compared with the corresponding adjacent normal tissues. $\mathrm{T}$, gastric cancer tissue; $\mathrm{N}$, matched non-tumorous gastric mucosa.

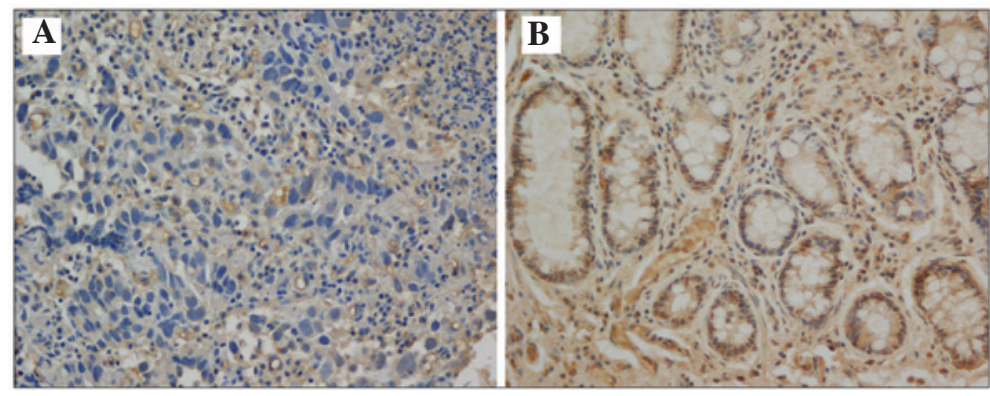

Figure 3. Protein expression of T-cadherin in (A) gastric cancer tissue and (B) surrounding normal tissue, as detected by immunohistochemistry with 3,3'-diaminobenzidine-tetrahydrochloride-dihydrate staining. Nuclei are stained blue with hematoxylin. The images demonstrated strong positive brown staining of T-cadherin expression in the cell membrane of gastric cancer cells and the adjacent normal tissue exhibited low expression levels. Magnification, $\mathrm{x} 400$.
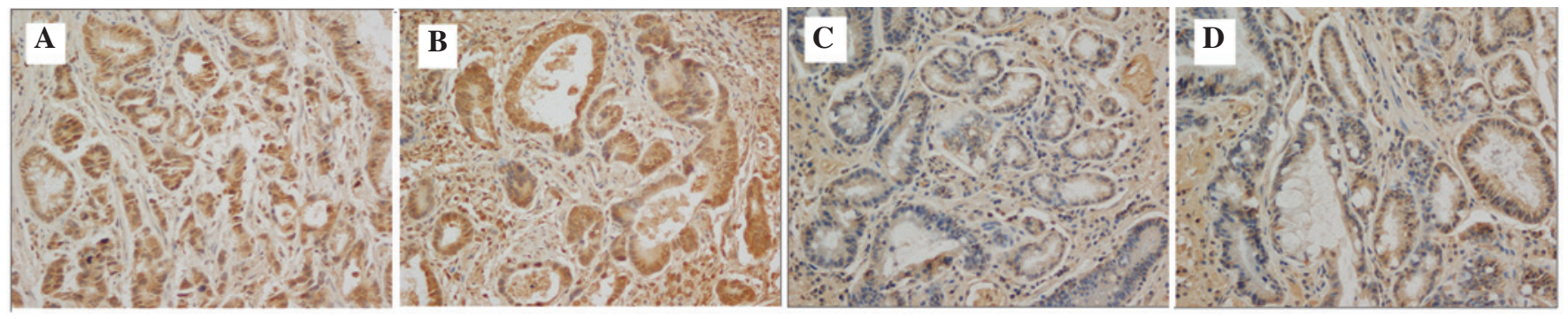

Figure 4. Correlation between the expression levels of T-cadherin and VEGF in gastric cancer tissues, detected by immunohistochemistry with 3,3'-diaminobenzidine-tetrahydrochloride-dihydrate staining. Nuclei are stained blue with hematoxylin. (A) Expression of T-cadherin in 'patient 1'. (B) Expression of VEGF in 'patient 1'. (C) Expression of T-cadherin in 'patient 2'. (D) Expression of VEGF in 'patient 2'. VEGF, vascular endothelial growth factor. The images demonstrated that the expression levels of VEGF were not associated with the expression of T cadherin. Magnification, $\mathrm{x} 400$.

between the protein expression levels of T-cadherin and various clinicopathological characteristics were assessed using a $\chi^{2}$ test. The overall survival curves were calculated using the Kaplan-Meier method and were analyzed using the log-rank test. A Cox Proportional-Hazards regression model was produced to determine which variables demonstrated individual prognostic value in determining patient survival rates. Cox regression analysis was performed at multivariate levels. $\mathrm{P}<0.05$ was considered to indicate a statistically significant difference and data are expressed as the mean \pm standard deviation.

\section{Results}

Expression levels of T-cadherin analyzed by RT-qPCR, western blotting and immunochemistry. The mRNA expression levels of T-cadherin in the tumor tissues and paired adjacent normal tissues were detected by RT-qPCR. The protein expression levels of T-cadherin were detected by western blotting and immunochemistry. The mRNA and protein expression levels of T-cadherin were significantly reduced in the gastric cancer tissue samples compared with the adjacent normal tissue samples (Figs. 1-3).

Clinical and pathological significance of the expression of T-cadherin in gastric cancer. The present study demonstrated that T-cadherin was expressed in the cell membrane and that the expression levels varied in the gastric cancer tissue and the adjacent normal tissue samples (Fig. 3). Among the 166 gastric cancer samples, 87 samples exhibited high expression levels, whereas the remaining 81 cases exhibited low expression levels (Table I). The adjacent normal tissue samples revealed the most marked levels of T-cadherin positive staining (Fig. 3). Decreased protein expression levels of T-cadherin were found to correlate with smoking, large tumor size (diameter $>4 \mathrm{~cm}$ ), lymph node metastasis and a higher TNM stage $(\mathrm{P}<0.05$ or $\mathrm{P}<0.01)$. However, the protein expression levels of $\mathrm{T}$-cadherin did not correlate with gender, age, alcohol intake, $H p$ infection or differentiation $(\mathrm{P}>0.05$; Table I).

Correlation between the expression levels of T-cadherin and $V E G F$. The present study determined the expression levels of 
Table II. Correlation between the expression levels of T-cadherin and VEGF in gastric cancer.

\begin{tabular}{lcccc}
\hline & \multicolumn{2}{c}{ T-cadherin } & \\
\cline { 2 - 4 } VEGF & Low & High & r-value & P-value \\
\hline Low & 64 & 29 & -0.084 & 0.283 \\
High & 102 & 55 & \\
\hline
\end{tabular}

VEGF, vascular endothelial growth factor.

Table III. Cox regression analysis of the association between clinicopathological variables, expression of T-cadherin and patient survival rates in gastric cancer.

\begin{tabular}{lll}
\hline Variable & HR $(95 \%$ CI & P-value \\
\hline Gender (male/female) & $0.943(0.535-1.663)$ & 0.839 \\
Age (<60 or $\geq 60$ years) & $1.005(0.649-1.556)$ & 0.983 \\
Smoking (no/yes) & $1.923(1.064-3.475)$ & 0.030 \\
Alcohol (no/yes) & $0.644(0.360-1.152)$ & 0.138 \\
Hp infection (no/yes) & $0.877(0.505-1.523)$ & 0.640 \\
Tumor size ( $\leq 4 \mathrm{~cm} />4$ cm) & $1.761(1.080-2.872)$ & 0.023 \\
Differentiation (well or moderate/poorly) & $2.094(1.257-3.488)$ & 0.005 \\
Histopathological (intestinal/diffuse) & $0.840(0.529-1.333)$ & 0.460 \\
Lymph node metastasis (no/yes) & $2.121(1.176-3.825)$ & 0.012 \\
TNM stages (I-II/III-IV) & $2.159(1.293-3.605)$ & 0.003 \\
T-cadherin expression (low/high) & $0.384(0.230-0.640)$ & 0.000 \\
\hline
\end{tabular}

Hp, Helicobacter pylori; TNM, tumor-node-metastasis; HR, hazard ratio; CI, confidence interval.

VEGF using immunochemistry in the tissue samples from 166 cases of gastric cancer. The result revealed that the expression levels of VEGF were not associated with the expression of T-cadherin (P>0.05; Fig. 4, Table II).

Correlation between the expression of T-cadherin and the overall survival rates of patients with gastric cancer. The patients with high expression levels of T-cadherin in their tissue sample had a significantly improved overall survival rate compared with patients exhibiting low expression levels of T-cadherin $(\mathrm{P}<0.01$; Fig. 5). The multivariate Cox regression analysis demonstrated that smoking, smaller tumor sizes (diameter $<4 \mathrm{~cm}$ ), no lymph node metastasis, good differentiation, a lower TNM stage and higher expression levels of T-cadherin were closely associated with a higher overall survival rate and were independent risk factors for gastric cancer $(\mathrm{P}<0.05$; Table III). However, the survival rate did not correlate with gender, age, alcohol intake, $H p$ infection or histopathological type $(\mathrm{P}>0.05)$.

\section{Discussion}

Previous studies have demonstrated that cancer progression is a multi-step process, in which cell-cell adhesion is important for the development of recurrent, invasive and distant metastasis (19). Multiple lines of evidence have indicated

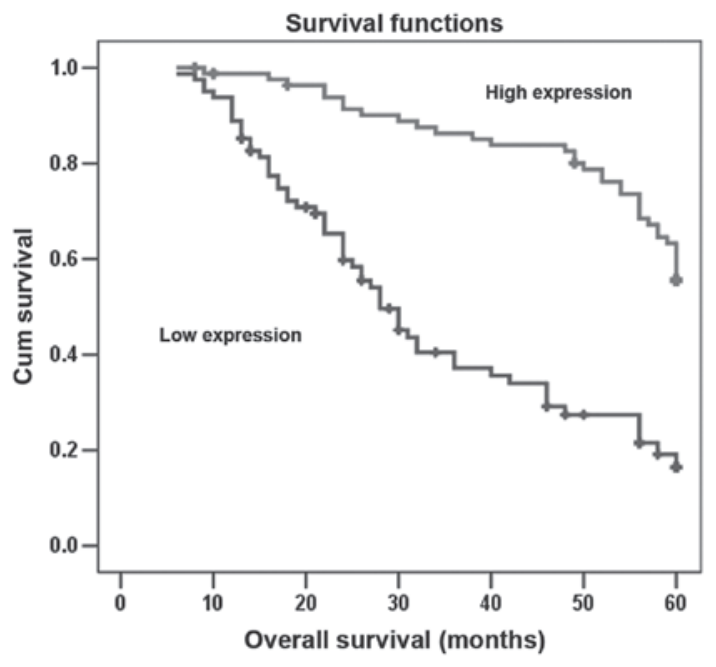

Figure 5. Kaplan-Meier survival curves demonstrating the overall survival rates of 166 patients with gastric cancer, and the association between surival rates and the expression of T-cadherin. The patients in the low T-cadherin expression group were found to have significantly lower survival rates compared with those in the high T-cadherin expression group (log-rank test, $\mathrm{P}<0.001)$. Cum, cumulative.

that alterations in the adhesion properties of cancer cells is vital in the development and progression of cancer. Loss of intercellular adhesion allows malignant cells to escape from 
their site of origin, degrade the extracellular matrix and acquire a more invasive and metastatic phenotype (20). Early evidence demonstrated certain adhesion molecules, which have been implicated in cancer as putative tumor suppressors, including E-cadherin (21). However, there certain adhesion molecules are also considered as pro-oncogenic proteins, including P-cadherin (22) and N-cadherin (23). CDH13, also termed T- or H-cadherin, is the only cadherin known to be membrane-anchored via a GPI anchor, rather than a transmembrane domain. The human $\mathrm{CDH} 13$ gene is often silenced in several types of cancer and it has long been considered to act against carcinogenesis (3). It has been suggested that T-cadherin may inhibit tumor progression, including proliferation, invasion and angiogenesis, through multiple pathways, including the Akt and SET7/9-p53 pathways $(8,24)$. However, only one previous study has investigated the correlation between the expression of T-cadherin and the clinicopathological features of gastric cancer, and this included a relatively small sample size (12).

Previous studies have revealed that the loss of T-cadherin is associated with methylation, and that treatment with a demethylating agent reactivates its expression $(6,7,25)$. The present study demonstrated that the T-cadherin protein was expressed in the cell membrane of the normal and the malignant gastric mucosa. The mRNA and protein expression levels of T-cadherin were reduced in the gastric cancer tissue samples compared with the corresponding normal tissue samples. In addition, its expression exhibited a close association with the clinicopathological features of gastric cancer. The decreased protein expression of T-cadherin was associated with a larger tumor size, lymph node metastasis and a higher TNM stage, however it was not associated with gender, age, alcohol intake differentiation or histopathological type. These results suggested that T-cadherin may be important in tumor growth, invasion and metastasis. This result was consistent with previous studies. Yan et al demonstrated an increased expression of T-cadherin and reduced cell proliferation in HepG2 cells (26), Philippova et al revealed that a loss of T-cadherin increases the metastatic potential and aggressiveness of squamous cell carcinoma (27) and Hebbard et al demonstrated that the loss of T-cadherin promotes tumor angiogenesis and metastasis in breast cancer (12). VEGF is the key mediator of angiogenesis and metastasis in cancer, and is upregulated by the expression of oncogenes $(28,29)$. Although the loss of T-cadherin has been considered to exhibit a prometastatic effect, the underlying mechanism remains to be elucidated. The present study assessed the expression of VEGF in gastric cancer and analyzed its association with the expression T-cadherin. The result demonstrated that the expression of VEGF was not associated with the expression of T-cadherin in the gastric cancer tissue samples, which suggested that other mechanism are responsible for prometastatic effects following loss of T-cadherin loss.

Liu et al indicated that T-cadherin may be used as an independent prognostic biomarker for bladder transitional cell carcinoma (30), wheras Kim et al demonstrated that T-cadherin may be used as a prognostic marker for patients with non-small cell lung cancer (10). In the present study, the survival rates of the patients revealed that those with high expression levels of T-cadherin had a significantly higher postoperative survival rate compared with those exhibiting low expression levels of T-cadherin. Therefore, patients with gastric cancer and reduced expression levels of T-cadherin may be a high-risk group with poor survival rates and may require more aggressive additional post-surgical systemic therapy. These results suggested that measurement of the protein expression levels of T-cadherin can assist in monitoring patient condition. According to the Cox regression analysis, a smaller tumor size, lack of lymph node metastasis, good level of differentiation, lower TNM stage and higher expression levels of T-cadherin were closely associated with increased overall survival rates, and the positive expression of T-cadherin appeared to be the most important independent prognostic predictor in gastric cancer.

Previous studies have demonstrated that smoking (31), alcohol consumption (32) and $H p$ infection (33) are risk factors for gastric cancer. Therefore, the present study investigated the possible associations between the expression of T-cadherin and smoking, alcohol and $H p$ infection. The results revealed that patients with a history of smoking exhibited low expression levels of T-cadherin. However, alcohol and $H p$ infection had no effect on the expression of T-cadherin. In addition, the survival rates of the patients suggested that those with a history of smoking had lower postoperative survival rates compared with the patients without a history of smoking, however, the overall survival rates were not associated with alcohol and $H p$ infection.

In conclusion, the expression of T-cadherin was found to decrease in gastric cancer and the expression levels were associated with smoking, tumor size, lymph node metastasis and TNM stage. The expression of T-cadherin may serve as an independent prognostic predictor and be used as a biomarker to predict the progression and prognosis of gastric cancer, however, no clear association was observed between the expression levels of VEGF and T-cadherin. In addition the results suggested that T-cadherin may exhibit a tumor suppressor function in gastric cancer and has the potential to be used as a target for therapeutic interventions for gastric cancer.

\section{Acknowledgements}

This study was supported by the National Natural Science Foundation of China (no. 81070328).

\section{References}

1. Jemal A, Bray F, Center MM, Ferlay J, Ward E and Forman D: Global cancer statistics. CA Cancer J Clin 61: 69-90, 2011.

2. Ali Z, Deng Y and Ma C: Progress of research in gastric cancer. J Nanosci Nanotechnol 12: 8241-8248, 2012.

3. Berx G and van Roy F: Involvement of members of the cadherin superfamily in cancer. Cold Spring Harb Perspect Biol 1: a003129, 2009.

4. Makrilia N, Kollias A, Manolopoulos L and Syrigos K: Cell adhesion molecules: role and clinical significance in cancer. Cancer Invest 27: 1023-1037, 2009.

5. Philippova M, Joshi MB, Kyriakakis E, Pfaff D, Erne P and Resink TJ: A guide and guard: the many faces of T-cadherin. Cell Signal 21: 1035-1044, 2009.

6. Chan DW, Lee JM, Chan PC and Ng IO: Genetic and epigenetic inactivation of T-cadherin in human hepatocellular carcinoma cells. Int J Cancer 123: 1043-1052, 2008.

7. Ren JZ and Huo JR: Correlation between T-cadherin gene expression and aberrant methylation of T-cadherin promoter in human colon carcinoma cells. Med Oncol 29: 915-918, 2012. 
8. Adachi Y, Takeuchi T, Nagayama T and Furihata M: T-cadherin modulates tumor-associated molecules in gallbladder cancer cells. Cancer Invest 28: 120-126, 2010.

9. Duan XS, Lu J, Ge ZH, Xing EH, Lu HT and Sun LX: Effects of T-cadherin expression on B16F10 melanoma cells. Oncol Lett 5: 1205-1210, 2013

10. Kim DS, Kim MJ, Lee JY, Kim YZ, Kim EJ and Park JY: Aberrant methylation of E-cadherin and $\mathrm{H}$-cadherin genes in nonsmall cell lung cancer and its relation to clinicopathologic features. Cancer 110: 2785-2792, 2007.

11. Toyooka KO, Toyooka S, Virmani AK, et al: Loss of expression and aberrant methylation of the $\mathrm{CDH} 13$ (H-cadherin) gene in breast and lung carcinomas. Cancer Res 61: 4556-4560, 2001.

12. Tang Y, Dai Y and Huo J: Decreased expression of T-cadherin is associated with gastric cancer prognosis. Hepatogastroenterology 59: 1294-1298, 2012.

13. Folkman J: Role of angiogenesis in tumor growth and metastasis. Semin Oncol 29: 15-18, 2002.

14. Saharinen P, Eklund L, Pulkki K, Bono P and Alitalo K: VEGF and angiopoietin signaling in tumor angiogenesis and metastasis. Trends Mol Med 17: 347-362, 2011.

15. Iurlova EI, Rubina KA, Sysoeva V, et al: T-cadherin suppresses the cell proliferation of mouse melanoma B16F10 and tumor angiogenesis in the model of the chorioallantoic membrane. Ontogenez 41: 261-270, 2010 (In Russian).

16. Hebbard LW, Garlatti M, Young LJ, Cardiff RD, Oshima RG and Ranscht B: T-cadherin supports angiogenesis and adiponectin association with the vasculature in a mouse mammary tumor model. Cancer Res 68: 1407-1416, 2008

17. Zhang J, Zhou Y and Jiang K: Evaluation of the seventh AJCC TNM staging system for gastric cancer: a meta-analysis of cohort studies. Tumour Biol 35: 8525-8532, 2014.

18. Livak KJ and Schmittgen TD: Analysis of relative gene expression data using real-time quantitative PCR and the 2-(-Delta Delta C(T)) Method. Methods 25: 402-408, 2001.

19. Okegawa T, Pong RC, Li Y and Hsieh JT: The role of cell adhesion molecule in cancer progression and its application in cancer therapy. Acta Biochim Pol 51: 445-457, 2004.

20. Zigler M, Dobroff AS and Bar-Eli M: Cell adhesion: implication in tumor progression. Minerva Med 101: 149-162, 2010.

21. Auerkari EI: Methylation of tumor suppressor genes p16 (INK4a), p27 (Kip1) and E-cadherin in carcinogenesis. Oral Oncol 42: $5-13,2006$
22. Albergaria A, Ribeiro AS, Vieira AF, et al: P-cadherin role in normal breast development and cancer. Int J Dev Biol 55: 811-822, 2011.

23. Mariotti A, Perotti A, Sessa C and Rüegg C: N-cadherin as a therapeutic target in cancer. Expert Opin Investig Drugs 16: 451-465, 2007.

24. Joshi MB, Ivanov D, Philippova M, Erne P and Resink TJ: Integrin-linked kinase is an essential mediator for T-cadherin-dependent signaling via Akt and GSK3beta in endothelial cells. FASEB J 21: 3083-3095, 2007.

25. Hibi K, Kodera Y, Ito K, Akiyama S and Nakao A: Methylation pattern of CDH13 gene in digestive tract cancers. Br J Cancer 91: 1139-1142, 2004

26. Yan Q, Zhang ZF, Chen XP, et al: Reduced T-cadherin expression and promoter methylation are associated with the development and progression of hepatocellular carcinoma. Int J Oncol 32: 1057-1063, 2008.

27. Philippova M, Pfaff D, Kyriakakis E, et al: T-cadherin loss promotes experimental metastasis of squamous cell carcinoma. Eur J Cancer 49: 2048-2058, 2013.

28. Welti J, Loges S, Dimmeler S and Carmeliet P: Recent molecular discoveries in angiogenesis and antiangiogenic therapies in cancer. J Clin Invest 123: 3190-3200, 2013.

29. Liu W, Xu J, Wang M, Wang Q, Bi Y and Han M: Tumor-derived vascular endothelial growth factor (VEGF)-a facilitates tumor metastasis through the VEGF-VEGFR1 signaling pathway. Int J Oncol 39: 1213-1220, 2011.

30. Lin YL, Liu XQ, Li WP, Sun G and Zhang CT: Promoter methylation of $\mathrm{H}$-cadherin is a potential biomarker in patients with bladder transitional cell carcinoma. Int Urol Nephrol 44: 111-117, 2012.

31. La Torre G, Chiaradia G, Gianfagna F, et al: Smoking status and gastric cancer risk: an updated meta-analysis of case-control studies published in the past ten years. Tumori 95: 13-22, 2009.

32. Duell EJ, Travier N, Lujan-Barroso L, et al: Alcohol consumption and gastric cancer risk in the European Prospective Investigation into Cancer and Nutrition (EPIC) cohort. Am J Clin Nutr 94: $1266-1275,2011$

33. Kato $\mathrm{M}$ and Asaka M: Recent knowledge of the relationship between Helicobacter pylori and gastric cancer and recent progress of gastroendoscopic diagnosis and treatment for gastric cancer. Jpn J Clin Oncol 40: 828-837, 2010 